\title{
Dexamethasone Enhances Surfactant Protein Gene Expression in Streptozotocin-Induced Immature Rat Lungs
}

\author{
HAMID H. RAYANI, JOSEPH B. WARSHAW, AND JOANNA FLOROS \\ Department of Cellular and Molecular Physiology, The Pennsylvania State University, College of \\ Medicine, Hershey, Pennsylvania [H.H.R., J.F.], and Department of Pediatrics, Yale University School of \\ Medicine, New Haven, Connecticut [J.B.W.]
}

\begin{abstract}
Because surfactant protein (SP) mRNA levels in rat fetuses are increased by maternal dexamethasone (dex) treatment and decreased in streptozotocin-induced diabetic (STZ-DB) pregnancy, we investigated the in vivo effects of dex on SP gene expression in STZ-DB pregnancy. The mRNA levels of SP (SP-A, SP-B, SP-C) were assessed in d 18 and 20 fetuses by Northern blot analysis, and nuclear run-on assays were performed with lung nuclei from d 20 fetuses (term $=22 \mathrm{~d}$ ). Our findings indicate: 1 ) dex causes a greater increase in SP-A and SP-B mRNA levels in d 18 (12-16-fold) compared with day 20 (4-6-fold) fetuses $(p<0.05)$ in normal and STZ-DB pregnancy; 2) a 2-3-fold increase in SP-C mRNA levels was observed in response to dex in d 18 and 20 fetuses; 3) the increase in transcription of SP-A and SP-B in d 20 fetuses after dex is 68 and $60 \%$, respectively, of the increase in their mRNA levels whereas
\end{abstract}

\section{ABSTRACT}

in STZ-DB, the decrease in transcription compared with mRNA levels is 3.67-fold for SP-A and 2.42 fold SP-B; and 4) changes in SP-C transcription in either in vivo model, dex-treated or STZ-DB, correspond well with changes in mRNA levels. Together, these findings indicate that dex can enhance SP expression in STZ-DB immature lungs and support differential regulation of fetal SP genes in the models studied. (Pediatr Res 38: 870-877, 1995)
SP, surfactant protein
Abbreviations
STZ, streptozotocin
STZ-DB, streptozotocin-induced diabetes
dex, dexamethasone
RDS, respiratory distress syndrome

Pulmonary surfactant is a lipoprotein complex that lines the air-liquid interface in the alveoli of the lungs. Surfactant is responsible for reducing surface tension at the air-liquid interface, thereby stabilizing the alveoli throughout the respiratory cycle and preventing their collapse at low lung volume. Infants born with insufficient amounts of surfactant fail to expand their lungs appropriately and may develop RDS (1). Pulmonary surfactant is composed predominantly of lipids (about 90\%) and about $5-10 \%$ proteins. There are, in addition to serum proteins (such as albumin), unique nonserum proteins called surfactant proteins (SPs). By convention, they are referred to as SP-A, -B, -C, and -D (2). SP-A is a $26-38-\mathrm{kD}$ sialoglycoprotein, SP-B and SP-C are two low molecular weight $(6-8-\mathrm{kD})$ hydrophobic proteins, and SP-D is a hydrophilic collagen-like protein. SP-A, SP-B, and SP-C play important roles in struc-

Received January 25, 1995; accepted July 3, 1995.

Correspondence: Joanna Floros, Ph.D., Department of Cellular and Molecular Physiology, The Pennsylvania State University, College of Medicine, 500 University Dr., Hershey, PA 17033.

Supported by the National Institutes of Health, RO1 HL38288 (J.F.), SCOR 5P50HL46488 (J.B.W.), and the American Heart Association, Pennsylvania Affiliate (H.H.R.). ture $(3,4)$, function (5-8), and metabolism (9) of surfactant. The role of SP-D in the function of surfactant is not yet defined (10).

Deficiency of pulmonary surfactant in prematurely born infants suggests lung immaturity. In humans, the lecithin: sphingomyelin ratio of $\geq 2$ and the presence of phosphatidylglycerol in amniotic fluid $(11,12)$ suggests fetal lung maturity. However, the infants in full term diabetic pregnancies are at increased risk of developing RDS, despite normal lecithin: sphingomyelin ratio of $\geq 2(13-15)$. Some reports have shown that the level of SP-A is reduced in amniotic fluid of pregnant diabetic women $(16,17)$, suggesting that decreased SP-A contributes to the increased incidence of RDS in infants of diabetic mothers. Comparable studies regarding the levels of SP-B and SP-C in amniotic fluid have not been reported. On the other hand, administration of glucocorticoids to pregnant women can increase fetal surfactant production and has been used clinically to prevent RDS in preterm infants $(18,19)$.

Glucocorticoids are shown to affect the expression of a small number of proteins in lung $(20,21)$, and some of these proteins are lung-specific (21). The effect of dex on SP-A gene expression in explant cultures varies in different species in a time- and 
dose-dependent manner, suggesting that regulation of SP-A is a complex process and that the mechanism is not completely conserved among species (22-25). However, the effect of dex on rat SP-A expression is found to be stimulatory both in vivo (26-29) and in vitro (25). In contrast to SP-A, all studies have demonstrated a stimulatory effect of glucocorticoids on SP-B and SP-C mRNA levels (28-31). Furthermore, we have also shown that transcription of SP-C is increased in dex-treated explants, (31) but no in vivo work has been reported. The mRNA levels of SP-D in rats also increase in response to glucocorticoid treatment, both in vitro and in vivo $(32,33)$. Similarly, SP-A, SP-B, and SP-C mRNA levels also increase developmentally during fetal life $(28,34,35)$. On the other hand, the levels of surfactant proteins (SP-A, SP-B) and the levels of SP mRNAs (SP-A, SP-B, SP-C) in rats are reduced $(36,37)$ in fetuses of STZ-DB pregnancy. These observations together suggest that a pretranslational event is involved in the regulation of SP gene expression in the two (STZ-DB and dex treatment) in vivo rat models. However, the mechanism by which dex and diabetes exert their effects in these models is not known.

In this report we have addressed two questions: 1) whether maternal dex treatment can enhance the SP mRNA levels in STZ-induced immature fetal lungs; and 2) whether maternal dex treatment and maternal STZ-DB exert their in vivo effects on SP mRNA levels by altering transcription.

\section{METHODS}

Animal model. Diabetes was induced in nonpregnant female Sprague-Dawley rats from Charles River Laboratories (Wilmington, MA) by i.v. injection of STZ, $50 \mathrm{mg} / \mathrm{kg}$ body weight (Sigma Chemical Co., St. Louis, MO) in $5 \mathrm{mM}$ sodium citrate in saline, $\mathrm{pH}$ 4.5. Diabetes was confirmed by blood glucose determination before mating and reconfirmed before sacrifice. Only females with blood glucose more than $250 \mathrm{mg} / \mathrm{dL}$ were used for the experiments. Conditions of mating and sacrifice have been previously described (38). Successful mating was confirmed by the presence of a copulatory plug or a vaginal smear positive for sperm on the morning after caging male and female rats together. The morning after mating occurred, is designated d 1 of gestation. Timed pregnant rats obtained from Charles River Breeding Laboratories were injected intraperitoneally with either $\operatorname{dex}(2 \mathrm{mg} / \mathrm{kg}$ body weight) or equivalent volume of DMSO, $24 \mathrm{~h}$ before sacrifice. At the same time, pregnant STZ-DB rats were injected either with dex $(2 \mathrm{mg} / \mathrm{kg}$ body weight) or equivalent volume of DMSO. The dex used had a concentration of $2 \mathrm{mg} / \mathrm{mL}$. Because our animals weighed between 250 and $350 \mathrm{~g}, 0.25-0.35 \mathrm{~mL}$ of either dex or DMSO was injected. The animals in all groups studied were killed 24 h later, either on d 18 or 20 of gestation, and fetuses were removed after hysterectomy. The fetal lungs were then frozen for RNA preparation. For d 18 and 20 fetuses, experiments were performed from 3 separate litters $(n=3)$. For $\mathrm{d} 18$, two to three fetuses from each litter were pooled for each analysis generating three separate pools of fetal lungs from each litter. These three samples from each litter were run on the same blot and the average of the values obtained from these three sam- ples were counted as one experiment $(n=1)$. Experiments from three litters $(n=3)$ were performed in this manner. For d 20 fetuses, lungs from each of the fetuses from any given litter were frozen separately. Lungs from three individual fetuses, each from a different litter $(n=3)$ were used for Northern analysis.

RNA preparation, blotting, and data analysis. Total lung RNA was prepared as described previously (26). Lung tissue was homogenized in a buffer containing $4 \mathrm{M}$ guanidium thiocyanate followed by centrifugation $(150000 \times g)$ of the homogenate through a cesium chloride cushion. The pelleted RNA was then resuspended, washed, and quantitated spectrophotometrically at $A_{260}$, and 20-30 $\mu \mathrm{g}$ of total RNA were subjected to $1 \%$ agarose-formaldehyde gel electrophoresis. The gel-fractionated RNA was then transferred to nylon membrane (Genescreen, DuPont NEN, Boston, MA). The RNA was immobilized by cross-linking with UV light at a setting of 120 $\mathrm{mJ}$ in UV Stratalinker 2400 (Stratagene, La Jolla, CA). The membrane was then hybridized with ${ }^{32} \mathrm{P}$-labeled cDNA using $1-3 \times 10^{5} \mathrm{cpm} / \mathrm{mL}$ (specific activity, $10^{7}-10^{8} \mathrm{cpm} / \mu \mathrm{g}$ ). After hybridization, the membranes were washed and exposed to XAR Kodak film. The membranes were stripped in a solution containing $50 \%$ deionized formamide and $1 \times \mathrm{SSC}$ for $2 \mathrm{~h}$ at $70^{\circ} \mathrm{C}$ and reprobed successively with SP-A, SP-B, SP-C, and actin cDNAs. The RNA bands on the film were quantified by soft laser densitometry. For SP-A, the sums of the densitometric values of both transcripts $(0.9$ and $1.6 \mathrm{~kb})$ were used for evaluation. The RNA level is expressed as the ratio of the densitometric value from each group hybridized to surfactant protein cDNA probe, to the densitometric value of the actin cDNA hybridization. Previously, we had shown that actin mRNA levels do not change in these models either developmentally or in response to either dex treatment (26) or diabetes (36). Actin cDNA hybridization is, therefore, used as an internal standard.

Transcription elongation assays. Methods for the isolation of nuclei, transcription elongation (nuclear run-on) assays, and isolation of ${ }^{32} \mathrm{P}$-labeled RNA were essentially as described by Veletza et al. (31). Nuclei were prepared from lungs of d 20 fetuses from control, dex-treated, and STZ-DB rats. Each transcription assay contained $2 \times 10^{7}$ nuclei in $200 \mu \mathrm{L}$ of reaction volume. Equivalent trichloroacetic acid-precipitable counts $\left({ }^{32} \mathrm{P}\right.$-labeled RNA to similar specific activity) from each group (controls, dex treatment, STZ-DB) were hybridized at $45^{\circ} \mathrm{C}$ for $3-5 \mathrm{~d}$ to separate slot blots each containing $5 \mu \mathrm{g}$ of linearized recombinant SP cDNAs, actin cDNA, and vector cDNA (SP65). Vector cDNA served as a negative control and actin cDNA served as a positive control. Hybridization was carried out in $46 \%$ deionized formamide, $2 \times$ Denhart's solution, $4 \times \mathrm{SSC}, 50 \mathrm{mM}$ 1,4-piperazinediethanesulfonic acid (PIPES) $\mathrm{pH} 7.0,2$ mM EDTA, pH 8.0, 0.1\% SDS, and 200 $\mu \mathrm{g} / \mathrm{mL}$ of yeast tRNA. After hybridization, filters were initially washed in $300 \mathrm{mM} \mathrm{NaCl}, 2 \mathrm{mM}$ EDTA, $10 \mathrm{mM}$ Tris, pH 7.8, for $30 \mathrm{~min}$ at $45^{\circ} \mathrm{C}$, then $30 \mathrm{~min}$ at $37^{\circ} \mathrm{C}$ in $25 \mathrm{~mL}$ of $300 \mathrm{mM}$ $\mathrm{NaCl}, 2 \mathrm{mM}$ EDTA, Tris, $\mathrm{pH} 7.8$, containing $175 \mu \mathrm{L}$ of RNase $\mathrm{T} 1\left(1 \times 10^{6} \mathrm{U} / \mathrm{mL}\right)$ and $50 \mu \mathrm{L}$ of RNase A $(10 \mathrm{mg} / \mathrm{mL})$. The final wash was performed for $30 \mathrm{~min}$ at $55^{\circ} \mathrm{C}$ in $300 \mathrm{mM} \mathrm{NaCl}$, $2 \mathrm{mM}$ EDTA, $10 \mathrm{mM}$ Tris, $\mathrm{pH} \mathrm{7.8,0.1 \%} \mathrm{SDS,} \mathrm{and} \mathrm{was}$ 
repeated three more times. The blots from each group of each experiment were processed simultaneously and exposed to the same Kodak XAR sheet of film with intensifying screens. The intensity of each band in resulting autoradiograms was quantitated by soft laser densitometer, and the relative levels of transcription for each SP gene were determined using the actin cDNA hybridization as an internal standard, as described above. Despite our efforts to increase the intensity of the SP-B hybridization signal, this signal remained weak and difficult to reproduce by photography. We tried to increase the intensity of the SP-B hybridization by using twice the number of nuclei and two labeled nucleotides. The failure to obtain a strong SP-B hybridization may in part be accounted for by delayed SP-B expression during development (34).

Statistical analyses. Densitometric values from Northern blots reflecting the levels of SP-A, SP-B, and SP-C mRNA were analyzed statistically after normalization to actin values. The ratios of with to without dex treatment were calculated in both groups of animals (normal and STZ-DB pregnancy). In each case, the densitometric values were derived from the same autoradiograph. In a similar manner, ratios of values obtained for STZ-DB to normal pregnancy (without dex treatment) were also calculated. All the values for mRNA levels in different groups of animals were also compared with controls (without dex treatment $)=1.00$. Densitometric values from transcription elongation assays were also analyzed. The ratios of values obtained for with to without dex treatment in normal pregnancy were calculated as well as the ratios of STZ-DB to normal pregnancy (without dex treatment) were calculated and compared with corresponding ratios of mRNA levels as described for Northern blots. Statistical analysis was performed by analysis of variance with Dunnett multiple comparison test. A $p$ value of less than 0.05 was taken to indicate a significant difference for the particular set of data.

\section{RESULTS}

Effects of maternal dex treatment on the mRNA levels of $S P$ in fetuses of normal and STZ-DB pregnancy. Figures 1 and 2 depict the effects of maternal dex treatment on mRNA levels of SP on normal and STZ-DB pregnancy in fetal d 18 and 20 , respectively. As previously reported $(36,37)$, the fetal mRNA levels of SP-A, SP-B, and SP-C are decreased in STZ-DB pregnancy (lanes 7-9) compared with normal pregnancy without dex treatment (lanes $4-6$ ). We have also reported that in vivo dex treatment enhances fetal mRNA levels of SP-A and SP-B in d 18-21 fetuses in normal pregnancy (28). In this report, we confirm these findings for gestational age 18 and 20 (lanes 1-6). In addition, we find that 1) mRNA levels of SP-C are enhanced in gestational d 18 and 20 (lanes $1-6)$ in response to maternal dex treatment; and 2) the fetal mRNA levels of SP-A, SP-B, and SP-C are enhanced in STZ-DB pregnancy within $24 \mathrm{~h}$ after maternal dex treatment in gestational d 18 and 20 (lanes 10-12) compared with those without dex treatment (lanes 7-9).

Quantitation of the surfactant protein mRNA levels in $\mathrm{d} 18$ and 20 fetuses of normal and STZ-DB pregnancy in the presence or absence of maternal dex treatment was carried out

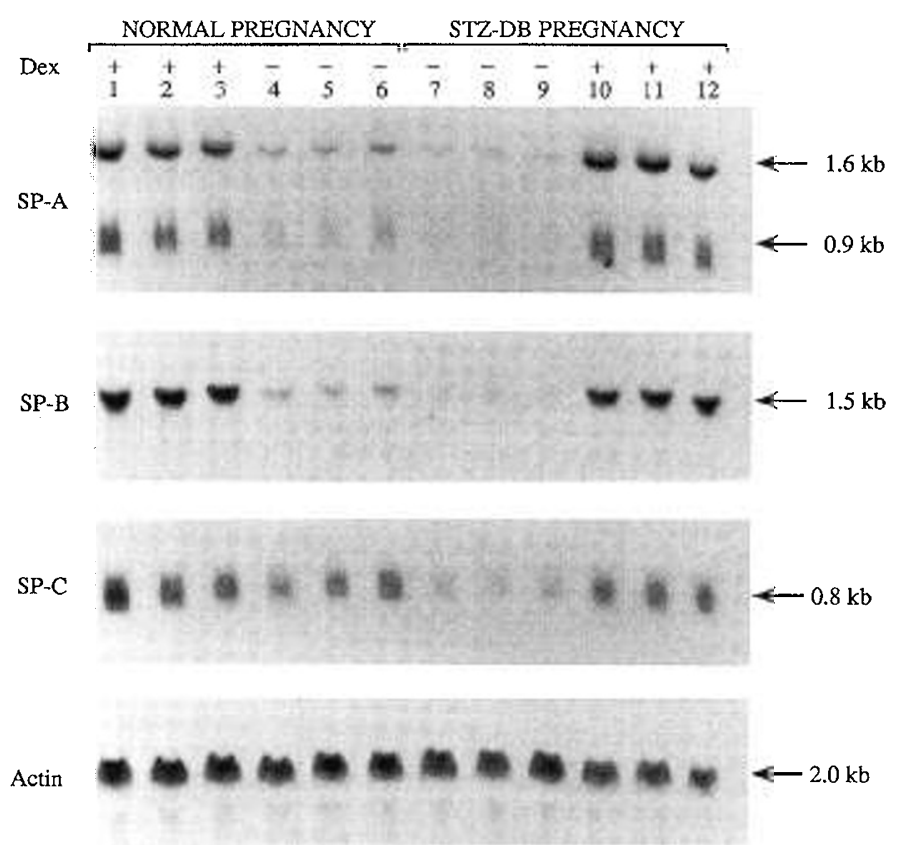

Figure 1. The mRNA levels of SP-A, SP-B, and SP-C in samples of total lung RNA from d 18 fetuses of normal and STZ-DB pregnancy with or without dex treatment. This figure is an autoradiographs of a representative RNA blot after hybridization, stripping, and reprobing with ${ }^{32} \mathrm{P}-$ labeled SP-A, SP-B, $\mathrm{SP}-\mathrm{C}$, and actin (positive control) cDNA probes. Each lane contains $30 \mu \mathrm{g}$ of total RNA from fetuses killed on d 18 of gestation. Samples from normal pregnancy are in lanes 1-3 with dex treatment and lanes 4-6 without dex treatment. Samples from STZ-DB pregnancy are in lanes 7-9 without dex treatment and lanes 10-12 with dex treatment. SP-A cDNA probe hybridizes to two SP-A messages of 1.6 and $0.9 \mathrm{~kb}$ in size, SP-B cDNA probe hybridizes to a $1.5-\mathrm{kb}$ message, SP-C cDNA probe to a $0.8-\mathrm{kb}$ SP-C message, whereas the actin cDNA probe hybridizes to $2.0-\mathrm{kb}$ message.

by densitometric analysis, and the results are depicted in Figure 3 (mean \pm SEM). We made three observations for the effects of maternal dex treatment on lungs of fetuses from normal and STZ-DB pregnancy. At first, the increase in fetal mRNA levels of SP-A and SP-B after maternal dex treatment in normal pregnancy in $\mathrm{d} 18$ fetuses is significantly higher $(p<0.05)$ compared with that seen in d 20 fetuses (Fig. 3A). After maternal dex treatment, the mRNA level of SP-A increased $15.83 \pm 5.25$-fold versus control in d 18 fetuses. This increase was considerably less in d 20 fetuses $(3.87 \pm 0.38$-fold versus control). In a similar way, mRNA levels of SP-B increased $13.80 \pm 3.25$-fold versus control in d 18 fetuses after maternal dex treatment whereas in $\mathrm{d} 20$ fetuses the increase was $4.90 \pm$ 0.41 -fold. Our second finding indicates that the increase in fetal mRNA levels of SP-A and SP-B after maternal dex treatment in STZ-DB pregnancy, as in control pregnancy, is much greater $(p<0.01)$ in $\mathrm{d} 18$ fetuses compared with that seen in $\mathrm{d} 20$ fetuses (Fig. 3B). The mRNA level of SP-A increased $19.07 \pm$ 4.80 -fold versus control in d 18 fetuses after maternal dex treatment compared with $2.40 \pm 0.10$-fold versus control in $\mathrm{d}$ 20 fetuses. In a similar manner, the SP-B mRNA level increased $12.60 \pm 1.25$-fold in 18 fetuses compared with a 5.86 \pm 0.88 -fold increase in $\mathrm{d} 20$ fetuses. The third finding indicates that the mRNA level of SP-C increased $\sim 2-3$-fold after maternal dex treatment in both groups, normal (Fig. $3 A$ ) as well as STZ-DB (Fig $3 B$ ) pregnancy. The SP-C increase was consid- 

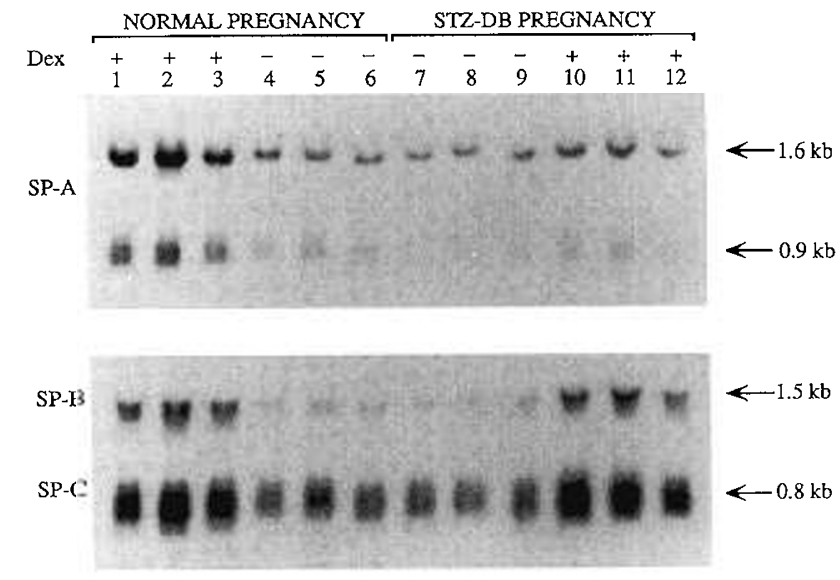

Actirt

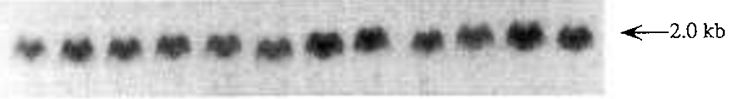

Figure 2. The mRNA levels of SP-A, SP-B, and SP-C in samples of total lung RNA from $\mathrm{d} 20$ fetuses of normal and STZ-DB pregnancy with or without dex treatment. This figure represents an autoradiograph of a single RNA blot after hybridization, stripping, and reprobing with ${ }^{32} \mathrm{P}-$ labeled SP-A, SP-B, SP-C, and actin (positive control) cDNA probes. Each lane contains $20 \mu \mathrm{g}$ of total RNA from fetuses killed on d 20 of gestation. Samples from normal pregnancy are in lanes 1-3 with dex treatment and lanes 4-6 without dex treatment. Samples from STZ-DB pregnancy are in lanes 7-9 without dex treatment and lanes 10-12 with dex treatment. SP-A cDNA probe hybridizes to two SP-A messages of 1.6 and $0.9 \mathrm{~kb}$ in size, SP-B cDNA probe hybridizes to a $1.5-\mathrm{kb}$ message, SP-C cDNA probe to a $0.8-\mathrm{kb}$ SP-C message, whereas the actin $\mathrm{cDNA}$ probe hybridizes to a $2.0-\mathrm{kb}$ message.

erably less than that seen for SP-A and SP-B in d 18 and 20 fetuses. Moreover, the difference in enhancement of SP-C mRNA levels after maternal dex treatment was not significantly different $(p>0.05)$ between 18 and 20 fetuses.

Evaluation of the effects of STZ-induced fetal lung immaturity on SP expression indicates that the mRNA levels of SP-A, SP-B, and SP-C are decreased in STZ-DB pregnancy compared with control pregnancy in $\mathrm{d} 18$ and 20 fetuses (Fig. $3 C$ ). The decrease is greatest for SP-A (d $18=0.466 \pm 0.10$; $\mathrm{d} 20=0.459 \pm 0.04)$ and least for SP-C $(\mathrm{d} 18=0.609 \pm 0.08$; $\mathrm{d} 20=0.647 \pm 0.06)$. The decrease in SP-B mRNA levels is $0.508 \pm 0.11$ for $\mathrm{d} 18$ and $0.587 \pm 0.09$ for $\mathrm{d} 20$ fetuses. The decrease in all SP mRNAs for d 18 fetuses is not significantly different $(p>0.05)$ from that seen in d 20 fetuses (Fig. 3C). Finally, the degree to which dex enhances SP mRNA levels in normal or STZ-DB pregnancy is not significantly different $(p$ $>0.05$ ) between the two groups, as shown by the ratio of with to without dex treatment (Fig. 3, $A$ and $B$ ). Inasmuch as the initial SP mRNA levels in STZ-DB pregnancy are lower when compared with those in normal pregnancy (Figs. $3 C$ and 4 ), the absolute values of mRNA levels after dex treatment in STZ-DB pregnancy do not rise to the levels seen in normal pregnancy in either gestational d 18 or 20 (Fig 4). The increase in SP-A mRNA levels after dex treatment in STZ-DB pregnancy were not significantly different in d 20 fetuses (Fig. 4B). This result could be partly due to rapid developmental increase seen around gestational d 19 (36).

Effect of dex or STZ-DB on transcription of surfactant proteins. To determine whether the changes observed in fetal
A. NORMAL PREGNANCY

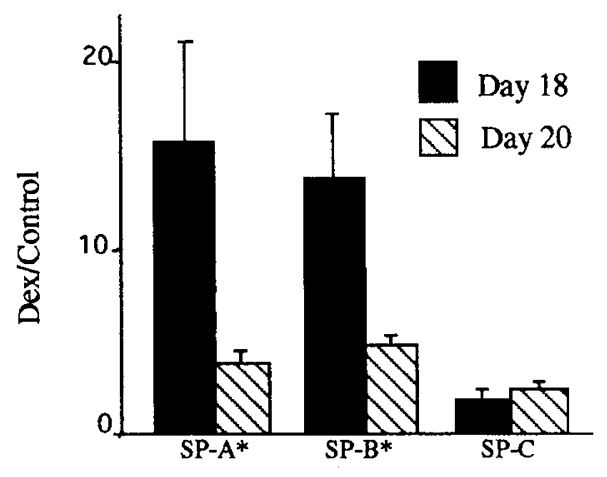

B. STZ-DB PREGNANCY

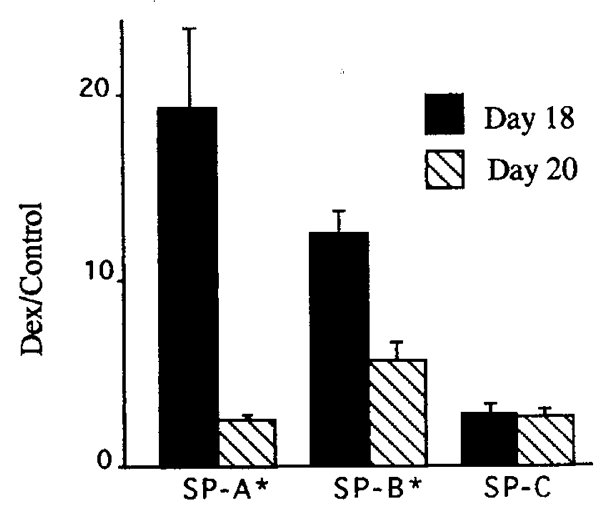

C. NO DEX TREATMENT

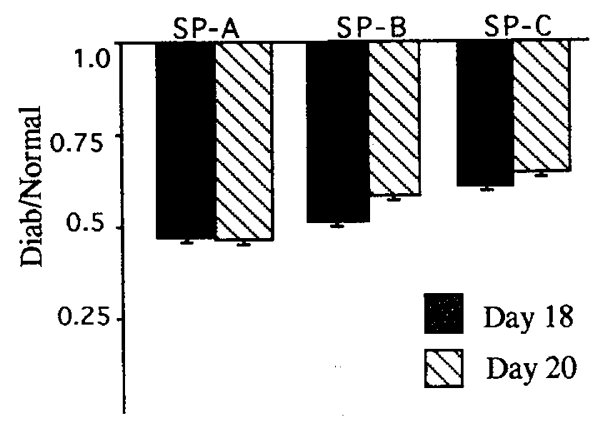

Figure 3. Diagram represents relative amounts of SP mRNAs in $d 18$ and $d$ 20 fetuses under various conditions. For $\mathrm{d} 18$ fetuses, data were obtained from three blots such as the one shown in Fig. $1(n=3)$, whereas for d 20 fetuses, data were obtained from one blot (Fig. 2) containing samples from three different litters $(n=3)$ in each of the four groups (see "Methods"). (A) The ratio of fetal mRNAs with to without dex treatment in normal pregnancy. $(B)$ Similar ratios in STZ-DB pregnancy. (C) The ratio of fetal mRNAs in STZ-DB to normal pregnancy without dex treatment. The asterisk $(*)$ represents significant ratios (Dex/Control) between $\mathrm{d} 18$ and d 20 fetuses with $p<0.05$. Values are expressed as mean \pm SEM.

mRNA levels of SP genes in response to STZ-DB and maternal dex treatment are due to alteration in transcription, we performed transcription elongation (nuclear run-on) assays. These assays were done using nuclei prepared from d 20 fetal lungs. Figure $5 A$ depicts the results of a representative transcription assay that shows decrease in transcription of SP-A and SP-C in STZ-DB compared with controls. The transcription of SP-B was also found to be decreased in STZ-DB (not shown, see "Methods"). Quantitation by densitometric analysis was car- 


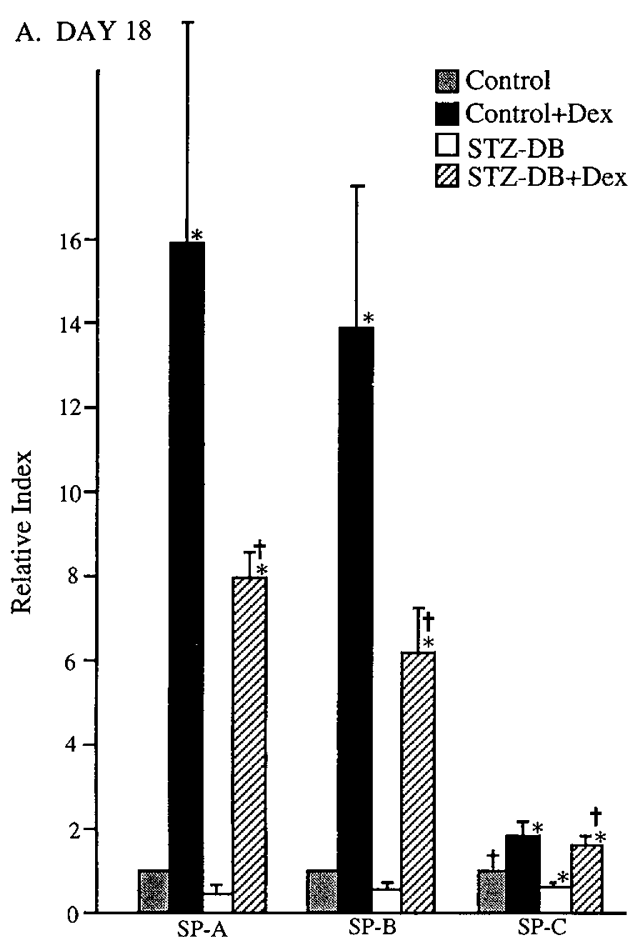

B. DAY 20

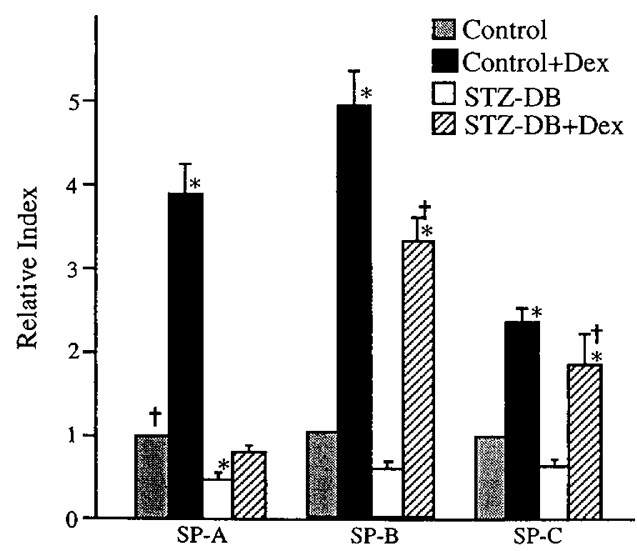

Figure 4. Relative abundance of mRNA for SP-A, SP-B, and SP-C under different conditions in rat fetuses from d $18(A)$ and $20(B)$ of gestation. The signal obtained by Northern hybridization was quantified densitometrically. The mean value for control is arbitrarily defined as 1.0 relative index. The asterisk $\left(^{*}\right)$ indicates values that are significantly different $(p<0.01)$ from control, and the dagger $(\dagger)$ indicates values that are significantly different $(p<$ 0.01) from STZ-DB. The error bars represent SEM.

ried out and the results are depicted in Figure $5 B$ (mean \pm SEM) along with the corresponding mRNA levels (mean \pm SEM) of SP seen in d 20 fetuses from STZ-DB. The relative decrease in transcription is greatest for SP-A $(0.125 \pm 0.02, n$ $=4)$ and least for SP-C $(0.679 \pm 0.07, n=5)$. The decrease in transcription for SP-B in STZ-DB is $(0.242 \pm 0.04, n=3)$. When we compared the extent of decrease in transcription to that of the mRNA levels, we observed that the decrease in transcription for SP-A and SP-B is significantly greater $(p<$ $0.05)$ than that observed for SP-A $(0.400 \pm 0.01, n=3)$ and SP-B $(0.587 \pm 0.09, n=3)$ mRNA levels. For SP-C, however,
A.

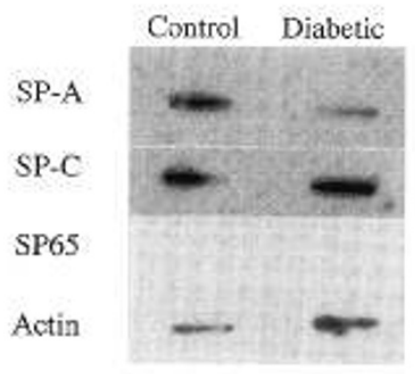

B.

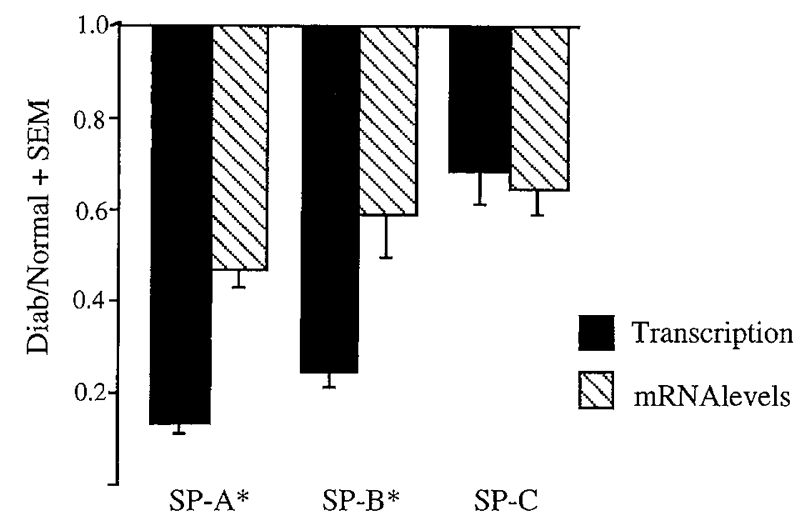

Figure 5. Transcription elongation (nuclear run-on) assay for SP and actin in nuclei from lungs of $\mathrm{d} 20$ fetuses from control and STZ-DB pregnancy. (A) A representative autoradiograph of transcription elongation assays where $5 \mu \mathrm{g}$ of linearized rat SP-A, SP-C, actin (positive control), and SP65 (a vector, negative control) cDNAs were applied in each slot as shown. The slot blots were then hybridized with ${ }^{32} \mathrm{P}$-labeled nascent transcripts from fetal lung nuclei from either control or STZ-DB pregnancy. $(B)$ The ratios of diabetic to control for transcription elongation assays and mRNA levels of SP-A, SP-B (see "Methods"), and SP-C. The asterisk $\left({ }^{*}\right)$ represent ratios (Diab/Normal) that are significantly different between transcription and mRNA levels with $p$ $<0.05$. Values are expressed as mean \pm SEM.

no significant difference $(p>0.05)$ was detected between the decrease in transcription $(0.679 \pm 0.07, n=5)$ and the decrease in the mRNA levels $(0.648 \pm 0.07, n=3)$.

Figure $6 A$ is a representative transcription assay and shows that transcription of SP-A and SP-C in d 20 fetal lungs is increased after maternal dex treatment. The transcription of SP-B was also found to be increased after dex treatment (not shown, see "Methods"). The data obtained after densitometric analysis (Fig. $6 B$ ) show that the mean \pm SEM for the relative enhancement of transcription of SP in response to dex treatment is $2.65 \pm 0.38(n=4)$ for SP-A, $2.91 \pm 0.62(n=3)$ for SP-B, and $1.95 \pm 0.46(n=5)$ for SP-C (Fig. 6B). The increase in transcription of SP-C compared with the increase in the corresponding mRNA levels $(2.36 \pm 0.13, n=3)$ after maternal dex treatment is not significantly different $(p>0.05)$. Although there is no statistically significant difference between transcription and corresponding mRNA levels for SP-A and SP-B, the relative increase in transcription of SP-A $(2.65 \pm$ $0.38, n=4)$ and SP-B $(2.91 \pm 0.62, n=3)$ seems to be less 
A.

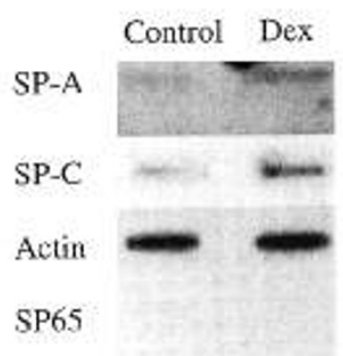

B.

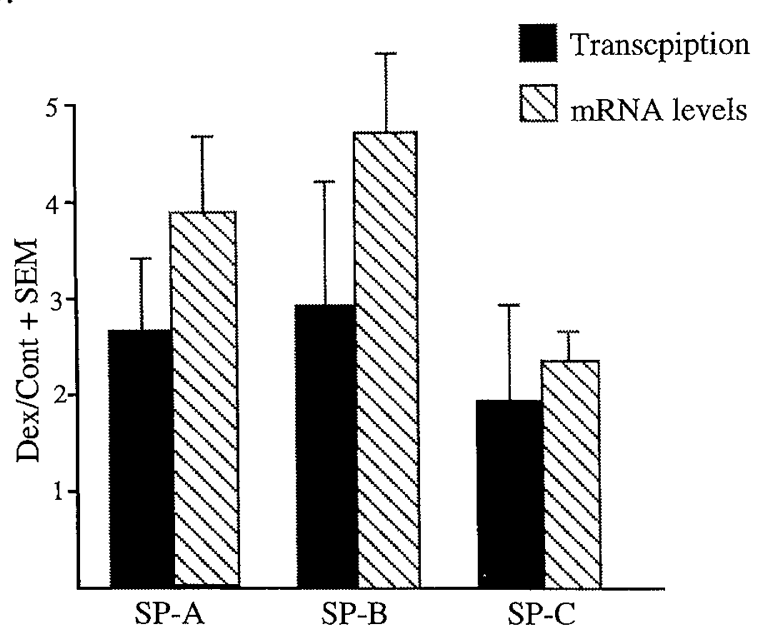

Figure 6. Transcription elongation (nuclear run-on) assay for SP and actin in nuclei from lungs of $\mathrm{d} 20$ fetuses from control and dex treated normal pregnancy. (A) A representative autoradiograph of transcription elongation assays where $5 \mu \mathrm{g}$ of linearized rat SP-A, SP-C, actin (positive control), and SP65 (a vector, negative control) CDNAs were applied in each slot as shown. The slot blots were then hybridized with ${ }^{32} \mathrm{P}$-labeled nascent transcripts from fetal lung nuclei from either control or dex treated normal pregnancy. $(B)$ The ratios of dex treatment to control for transcription elongation assays and mRNA levels of SP-A, SP-B (see "Methods"), and SP-C. Values are expressed as mean $\pm \mathrm{SEM}$.

than the increase in mRNA levels of SP-A $(3.87 \pm 0.38, n=$ 3) and SP-B $(4.71 \pm 0.41, n=3)$.

Effect of maternal dex treatment and STZ-DB on fetal weight and lung weight. The effect of maternal dex (28) treatment and STZ-DB (36) on fetal weight and lung weight were similar to those seen earlier. There was a decrease in fetal and lung weights after dex treatment in normal pregnancy, as reported earlier (28) as well as in STZ-DB pregnancy compared with their respective controls. There was also a decrease in fetal and lung weight in STZ-DB compared with normal pregnancy (without dex treatment) as reported earlier (36).

\section{DISCUSSION}

Our results show that maternal dex treatment enhances the SP mRNA levels in the STZ-induced immature fetal lungs, suggesting that STZ-DB does not block the stimulatory effects of dex inspite of the opposite effects that STZ-DB and maternal dex have on SP mRNA levels. Although the magnitude of the dex-enhanced SP mRNA levels is similar in both groups (normal and STZ-DB pregnancy), the dex-enhanced SP mRNA levels in fetuses of STZ-DB mothers do not reach the levels seen in controls after dex treatment. Because SP mRNA levels are lower after dex treatment in STZ-DB compared with normal pregnancy, the above observation suggests that dex treatment cannot overcome the inhibitory effect that STZ-DB has on basal SP mRNA levels. These findings further suggest that some characteristic differences exist in the mechanism(s) by which these two experimental models exert their effects on SP gene expression. Infants born to uncontrolled diabetic mothers are at increased risk for developing RDS (13-15) compared with their normal counterparts. Because SP-A (16, 17) is found to be reduced in amniotic fluid of pregnant diabetic women, it is possible that careful administration of dex to diabetic mothers before parturition could enhance expression of SP-A, and of other SP. These changes in turn may contribute to a reduction in the incidence of RDS in infants born to these mothers.

We detected SP-A, SP-B, and SP-C mRNA in d 18 rat fetuses, which is consistent with results obtained earlier in our laboratory $(28,36,37)$ but in contrast to findings of Schellhase et al. $(29,34)$ who did not detect SP-A and SP-B mRNAs on gestational age d 17. (Gestational d 17 in the reports by Schellhase and colleagues corresponds to $\mathrm{d} 18$ in our experiments due to differences in the definition of gestational d 1.) Moreover, SP-A mRNA levels have been detected as early as $16 \mathrm{~d}$ of gestation by primary translation products (27). More recently (39), SP-A, SP-B, and SP-C mRNA levels have been detected by reverse-transcriptase polymerase chain reaction, as early as $13 \mathrm{~d}$ of gestation. Furthermore, the effect of dex on mRNA levels of SP-A and SP-B, in both normal and STZ-DB pregnancy, is more pronounced in d 18 compared with $\mathrm{d} 20$ rat fetuses. The findings of the control pregnancy are consistent with previous findings $(28,29)$. It is possible that as the levels of SP mRNA increase with advancing gestational age $(28,34)$, dex treatment in the late stages of gestation has a limited role in enhancing SP mRNA levels. The dex-induced increase in SP-C mRNA, on the other hand, was smaller than that in SP-A and SP-B mRNA, and there was no significant difference in the degree of enhancement of SP-C mRNA between d 18 and 20 rat fetuses. These results may be explained by the earlier appearance of SP-C mRNA during fetal development compared with SP-A and SP-B mRNA (34). However, it is also likely that SP-C is involved in other important developmental functions and therefore, it may be regulated differently from SP-A and SP-B.

Several factors, including insulin and glucose, have been implicated in the inhibition of SP synthesis and SP mRNA levels in vitro $(40,41)$. Uncontrolled diabetic pregnancy in humans is accompanied by fetal hyperglycemia and hyperinsulinemia. The STZ-DB model used here, exhibits fetal hyperglycemia with normal to low plasma insulin levels $(38,42)$, suggesting that hyperglycemia has an important role in inhibiting SP mRNA levels in vivo. In this regard, high glucose $(50-100 \mathrm{mM})$ but not insulin $(1 \mathrm{U} / \mathrm{mL})$ delayed fetal lung development in $\mathrm{d} 20$ fetal rat lung explants as determined by 
reduced incorporation of ${ }^{3} \mathrm{H}$ into phosphatidylcholine and disaturated phosphatidylcholine (43). Furthermore, fetal lung phosphatidylcholine and disaturated phosphatidylcholine were increased to normal level in STZ-DB in d 21 and 22 rat fetuses by dex treatment, suggesting that delayed fetal lung maturation seen in STZ-DB could be reversed by dex (44). The levels of SP mRNAs were not examined in either of these studies (43, 44). Thus, hyperglycemia appears to be one of the factors involved in the regulation of lung maturation/fetal surfactant protein gene expression in STZ-DB pregnancy. In adult rats, the effect of the STZ-DB state on SP-A and SP-B (but not SP-C) mRNA levels $(45,46)$ is different from that observed in the fetal lung (36) suggesting that different mechanisms are operative in the regulation of SP expression in STZ-induced immature fetal lung and adult STZ-DB rats.

Transcription of all three SP is reduced in STZ-DB and enhanced by dex treatment in lungs of $\mathrm{d} 20$ fetuses. This is the first in vivo demonstration that a transcriptional step is involved in the regulation of surfactant protein gene expression in response to STZ-induced diabetes. However, the degree of inhibition of transcription among the three SP in STZ-DB differs, being highest for SP-A and lowest for SP-C. The degree of inhibition of transcription of SP-C in fetuses of STZ-DB compared with control corresponds well with reduction in their mRNA levels (Fig. $4 B$ ). It seems likely that the regulatory control for SP-C mRNA occurs primarily at the transcriptional level in the STZ-DB model. In contrast, inhibition of transcription of SP-A and SP-B in STZ-DB is significantly greater $(p<0.05)$ than the decrease observed in their corresponding mRNA levels (Fig. 4B), suggesting that in addition to a transcriptional control, posttranscriptional mechanism(s) are involved in SP-A and SP-B gene expression in the STZ-DB model. It is possible that reduction in the transcription of SP-A and SP-B is partially offset by an increase in mRNA stability. In this regard, $\mathrm{Na}$ butyrate has been shown to reduce SP-A and SP-B mRNA levels by affecting both transcription and mRNA stability, in explant rat lung cultures (47). Whether butyric acid analogs as $\beta$-hydroxybutyrate that is increased in diabetes can mediate the reduction in SP mRNAs observed in fetuses of STZ-DB pregnancy remains to be determined. Furthermore, the stability of several mRNAs is shown to increase in the presence of a transcription blocking drug. For example, actinomycin D increases transferrin receptor mRNA stability more than 10-fold (48). In this regard, the stability of SP-B mRNA in a human pulmonary adenocarcinoma cell line, NCIH441-4 cells is increased after actinomycin D treatment in the presence or absence of glucocorticoids (49). Additionally, in the same system, the inhibitory effects of TNF- $\alpha$ and TPA on SP-A and SP-B mRNA levels are blocked by actinomycin D (50). Whether STZ-DB acts similar to actinomycin D and affects transcription of factors involved in mRNA stability of SP-A and SP-B is currently unknown. Moreover, the SP-B mRNA levels were markedly reduced in NCI-H441-4 cell line after exposure to puromycin, an inhibitor of protein synthesis. Whether continuous protein synthesis is required for the stability of SP message in vivo is unknown.

The degree of increase in transcription of SP-A and SP-B in response to in vivo dex treatment may only partially account for the total content of SP-A and SP-B mRNA levels observed. The increase in the rate of transcription for SP-A and SP-B at gestational d 20 is 68 and $60 \%$, respectively, of the observed increase in the SP-A and SP-B mRNA levels. This observation suggests a role of posttranscriptional mechanism(s) in the regulation of SP-A and SP-B mRNA levels. Alternatively, the differences observed between transcription and mRNA content could be due to an accumulative effect as a result of increased synthesis with no change in the rate of mRNA degradation. However, previous in vitro studies in NCI-H441-4 cell culture system have shown that the dex-enhanced transcription of SP-B is only partially responsible for the increase in their mRNA levels (49) and in human fetal lung explants, glucocorticoids regulate SP-A (51) and SP-B (52) expression by affecting transcription as well as mRNA stability. In contrast to SP-A and SP-B, we found that the difference between changes in mRNA levels of SP-C and its transcription in response to dex treatment is not significantly different. These results are also consistent with in vitro findings in fetal rat lung explants where no significant differences between SP-C transcription and SP-C mRNA levels in response to dex treatment were detected (31). Moreover, Venkatesh et al. 1993 (52) found that enhancement of SP-C mRNA in response to glucocorticoids involves increased transcription without a change in mRNA stability in human fetal lung explants. Together these observations indicate that posttranscriptional mechanisms may not have any significant role in regulation of SP-C mRNA levels. This finding also suggests that SP-C mRNA is regulated differently from SP-A and SP-B mRNA in this respect.

In conclusion, we show that maternal dex treatment enhances SP mRNA levels in the STZ-induced immature fetal lungs suggesting that STZ-DB pregnancy does not prevent the stimulatory effects of dex on SP mRNA levels. Maternal dex treatment, however, does not appear to overcome the basal inhibition by STZ-DB. Furthermore, the changes seen in SP-A and SP-B mRNA levels in response to dex or STZ-DB are at least in part due to alteration in transcription of SP genes. The effect of dex or STZ-DB on SP-C mRNA appears to involve primarily a transcriptional control.

Acknowledgments. The authors thank Dr. D. S. Phelps for his critical input and help with animal models and Kris Snow for technical assistance.

\section{REFERENCES}

1. Avery ME, Mead J 1959 Surface properties in relation to atelectases and hyaline membrane disease. Am J Dis Child 97:517-523

2. Possmayer F 1988 A proposed nomenclature for pulmonary surfactant associated proteins. Am Rev Respir Dis 138:990-998

3. Suzuki Y, Fujita Y, Kogishi K 1989 Reconstitution of tubular myelin from synthetic lipids and proteins associated with pig pulmonary surfactant. Am Rev Respir Dis 140:75-81

4. Williams MC, Hawgood S, Hamilton RL 1991 Changes in lipid structure produced by SPs SP-A, SP-B and SP-C. Am J Respir Cell Mol Biol 5:41-50

5. Smith GH, Taeusch HW, Phelps DS, Keough KMW 1988 Mixtures of low molecular weight surfactant proteins and dipalmitoyl phosphatidylcholine duplicate effects of pulmonary surfactant in vitro and in vivo. Pediatr Res 23:484-490

6. Yu SH, Wallace D, Bhavnani B, Enhorning G, Harding PGR, Possmayer F 1988 Effects of reconstituted pulmonary surfactant containing the 6000-Dalton hydrophobic protein on lung compliance of prematurely delivered rabbit fetuses. Pediatr Res 23:23-30 
7. Hawgood S, Benson BJ, Hamilton Jr RJ 1985 Effects of a surfactant-associated protein and calcium ions on the structure and surface activity of lung surfactant lipids. Biochemistry 24:184-190

8. Whitsett JA, Hull WM, Ohning B, Ross G, Weaver TE, Holm BA, Shapiro DL, Notter RH 1986 Hydrophobic surfactant-associated protein in whole lung surfactant and its importance for biophysical activity in lung surfactant extracts used for replacement therapy. Pediatr Res 20:460-467

9. Wright JR, Clements JA 1987 Metabolism and turnover of surfactant. Am Rev Respir Dis $136: 426-444$

10. Persson A, Change D, Rust K, Moxley M, Longmore W 1989 Purification and biochemical characterization of CP4 (SP-D), a collagenous surfactant-associated protein. Biochemistry 28:6361-6367

11. Gluck L, Kulovich MV, Borer Jr RC, Keidel WWK 1974 The interpretation and significance of the lecithin/sphingomyelin ratios in amniotic fluid. Am J Obstet Gynecol 120:142-155

12. Torday J, Carson L, Lawson EE 1979 Saturated phosphatidylcholine in amniotic fluid and prediction of the respiratory-distress syndrome. N Engl I Med 301:1013-1018

13. Robert MF, Neff RK, Hubbell JP, Taeusch HW, Avery ME 1976 Association between maternal diabetes and the respiratory-distress syndrome in the newborn. $N$ Engl $J$ Med 294:357-360

14. Bourbon JR, Farrell PM 1985 Fetal lung development in the diabetic pregnancy. Pediatr Res 19:253-267

15. James DK, Chiswick ML, Harkes A, Williams M, Tindall VR 1984 Maternal diabetes and neonatal respiratory distress. I. Maturation of fetal surfactant. $\mathrm{Br} \mathrm{J}$ Obstet Gynaecol 91:316-324

16. Katyal SL, Amenta JS, Singh G, Silverman JA 1984 Deficient lung surfactant apoproteins in amniotic fluid with mature phospholipid profile from diabetic pregnancies. Am J Obstet Gynecol 148:48-53

17. Snyder JM, Kwan JE, O'Brien JA, Rosenfield, Odom MJ 1988 The concentration of the $35 \mathrm{kDa}$ surfactant apoprotein in amniotic fluid from normal and diabetic pregnancies. Pediatr Res 24:728-734

18. Liggins GC, Howie RN 1972 A controlled trial of antepartum glucocorticoid treatment for prevention of the respiratory distress syndrome in premature infants. Pediatrics 50:515-525

19. Morales WJ, Diebel D, Lazar AJ, Zadrozny D 1986 The effect of antenatal dexamethasone administration on the prevention of respiratory distress syndrome in preterm gestations with premature rupture of membranes. Am J Obstet Gynecol 154:591-595

20. Phelps DS, Giannopoulos G 1984 Effect of dexamethasone on the synthesis of specific proteins in fetal rabbit lung in vivo and in organ culture. Exp Lung Res 7:195-210

21. Floros J, Phelps DS, Smith BT 1985 Two dimensional gel electrophoresis of dexamethasone-induced organ-specific developmental proteins (fsa, fsb) in rat lung fibroblasts. Electrophoresis 6:238-241

22. Liley HG, White RT, Benson BJ, Ballard PL 1988 Glucocorticoids both stimulate and inhibit production of pulmonary surfactant protein $\mathrm{A}$ in fetal human lung. Proc Natl Acad Sci USA 85:9096-9100

23. Boggaram V, Smith ME, Mendelson CR 1989 Regulation of expression of the gene encoding the major surfactant protein (SP-A) in human fetal lung in vitro. J Biol Chem 264:11421-11427

24. Boggaram V, Mendelson CR 1988 Transcriptional regulation of the gene encoding the major surfactant protein (SP-A) in rabbit fetal lung. J Biol Chem 263:1906019065

25. Nichols VK, Floros J, Dynia DW, Veletza SV, Wilson CM, Gross I 1990 Regulation of surfactant protein A (SP-A) mRNA by hormones and butyrate in cultured fetal rat lung. Am J Physiol 259:L488-L495

26. Floros J, Phelps DS, Harding HP, Church S, Ware J 1989 Postnatal stimulation of rat surfactant protein A synthesis by dexamethasone. Am J Physiol 257:L137-L143

27. Phelps DS, Church S, Kourembanas S, Taeusch HW, Floros J 1987 Increases in the $35 \mathrm{kDa}$ surfactant -associated protein and its mRNA following in vivo dexamethasone treatment of fetal and neonatal rats. Electrophoresis 8:235-238

28. Phelps DS, Floros J 1991 Dexamethasone in vivo raises surfactant protein B mRNA in cultured fetal rat lung. Am J Physiol 260:L149 - L152
29. Schellhase DE, Shannon JM 1991 Effects of maternal dexamethasone on expression of SP-A, SP-B, and SP-C in the fetal rat lung. Am J Respir Cell Mol Biol 4:304-312

30. Floros J, Gross I, Nichols KV, Veletza SV, Dynia D, Lu H, Wilson CM, Peterec SM 1991 Hormonal effects on the surfactant protein B (SP-B) mRNA in alveolar and bronchiolar epithelium. Am J Respir Cell Mol Biol 4:449-454

31. Veletza SV, Nichols KV, Gross I, Lu H, Dynia DW, Floros J 1992 Surfactant protein C: Hormonal control of SP-C mRNA levels in vitro. Am J Physiol 262:L684-L687

32. Deterding RR, Shimizu H, Fisher JH, Shannon JM 1994 Regulation of the surfactant protein D expression by glucocorticoids in vitro and in vivo. Am J Respir Cell Mol Biol 10:30-37

33. Mariencheck W, Crouch E 1994 Modulation of surfactant protein D expression by glucocorticoids in fetal rat lung. Am J Respir Cell Mol Biol 10:419-429

34. Schellhase DE, Emrie PA, Fisher JH, Shannon JM 1989 Ontogeny of surfactant apoproteins in the rat. Pediatr Res 26:167-174

35. Ohashi T, Polk D, Ikegami M, Ueda T, Jobe A 1994 Ontogeny and effects of exogenous surfactant treatment on SP-A, SP-B, and SP-C mRNA expression in rabbit lungs. Am J Physiol 267:L46-L51

36. Guttentag SH, Phelps DS, Stenzel W, Warshaw JB, Floros J 1992 Surfactant protein-A expression is delayed in fetuses of streptozotocin treated rats. Am J Physiol 262:L489-L494

37. Guttentag SH, Phelps DS, Warshaw JB, Floros J 1992 Delayed hydrophobic surfactant protein (SP-B, SP-C) expression in fetuses of streptozotocin treated rats. Am J Respir Cell Mol Biol 7:190-197

38. Gewolb IH, Barrett C, Wilson DM, Warshaw JB 1982 Delay in pulmonary glycogen degradation in fetuses of streptozotocin diabetic rats. Pediatr Res 16:869-873

39. Jinxia W, Souza P, Kuliszewski M, Tanswell SK, Post M 1994 Expression of surfactant proteins in embryonic rat lung. Am J Respir Cell Mol Biol 10:222-229

40. Snyder JM, Mendelson CR 1987 Insulin inhibits the accumulation of the major lung surfactant apoprotein in human fetal lung explants maintained in vitro. Endocrinology $120: 1250-1257$

41. Dekowski SA, Snyder JM 1992 Insulin regulation of messenger ribonucleic acid for the surfactant-associated proteins in human fetal lung in vitro. Endocrinology 131:669-676

42. Bourbon JR, Pignol B, Marin L, Rieutort M, Tordet C 1985 Maturation of fetal rat lung in diabetic pregnancies of graduated severity. Diabetes 34:734-743

43. Gewolb IH 1993 High glucose causes delayed fetal lung maturation in vitro. Exp Lung Res 19:619-630

44. Rotenberg M, Gewolb IH 1993 Reversal of lung maturational delay in the fetus of the diabetic rat using triiodothyronine or dexamethasone. Biol Neonate 64:318-324

45. Sugahara K, Iyama K, Sano K, Morioka T 1992 Overexpression of pulmonary apoprotein A mRNA in alveolar type II cells and nonciliated bronchiolar (Clara) epithelial cells in streptozotocin-induced diabetic rats demonstrated by in situ hybridization. Am J Respir Cell Mol Biol 6:307-314

46. Sugahara K, Iyama K, Sano K, Morioka T 1994 Differential expression of surfactant protein SP-A, SP-B, and SP-C mRNAs in rats with streptozotocin-induced diabetes demonstrated by in situ hybridization. Am J Respir Cell Mol Biol 11:397-404

47. Peterec SM, Nichols KV, Dynia DW, Wilson CM, Gross I 1994 Butyrate modulates surfactant protein mRNA in fetal rat lung by altering mRNA transcription and stability. Am J Physiol 267:L9-L15

48. Mullner EW, Neupert B, Kuhn LC 1989 A specific mRNA-binding factor regulates the iron-dependent stability of cytoplasmic transferrin receptor mRNA. Cell 58:373382

49. O'Reilly MA, Clark JC, Whitsett JA 1991 Glucocorticoid enhance pulmonary surfactant protein B gene transcription. Am J Physiol 260:L37-L43

50. Whitsett JA, Clark JC, Wispe JR, Pryhuber GS 1992 Effects of TNF- $\alpha$ and phorbol ester on human surfactant protein and MnSOD gene transcription in vitro. Am J Physiol 262:L688-L693

51. Iannuzzi DM, Ertsey R, Ballard PL 1993 Biphasic glucocorticoid regulation of pulmonary SP-A: characterization of inhibitory process. Am J Physiol 264:L236L244

52. Venkatesh VC, Iannuzzi DM, Ertsey R, Ballard PL 1993 Differential glucocorticoid regulation of the pulmonary hydrophobic surfactant proteins SP-B and SP-C. Am J Respir Cell Mol Biol 8:222-228 